\section{Adaptação transcultural para o Brasil do instrumento PRISMA-7: avaliação das equivalências conceitual, de item e semântica}

\author{
Cross-cultural adaptation of the PRISMA-7 \\ instrument for use in Brazil: evaluation of \\ conceptual, item, and semantic equivalences
}

Ana Luiza Flores Saenger 1

Celia Pereira Caldas 1

Luciana Branco Motta 1

\title{
Resumo
}

O rastreamento é uma maneira eficaz de captar idosos em risco de declínio funcional. Para tanto, é necessário um instrumento simplificado, capaz de identificar esses indivíduos para uma avaliação posterior mais abrangente. Entretanto, a utilização de um instrumento de medida em

1 Universidade do Estado do Rio de Janeiro, Rio de Janeiro, Brasil.

\author{
Correspondência \\ A. L. F. Saenger \\ Rua Dois de Dezembro 78 , \\ Rio de Janeiro, $R J$ \\ 22220-040, Brasil. \\ analuizasaenger@gmail.com
} requer adaptação prévia. Este artigo tem por objetivo descrever as primei-
rento sociocultural diferente daquele para o qual foi desenvolvido, ras etapas do processo de adaptação transcultural do PRISMA-7, questionário desenvolvido para identificar idosos que vivem na comunidade e se encontram em risco de perda funcional. As avaliações das equivalências, conceitual e de itens mostraram pertinência no contexto brasileiro. A equivalência semântica contemplou a correspondência do significado referencial (denotativo) e do significado conotativo. O pré-teste demonstrou que a versão-síntese do instrumento tem boa aceitabilidade. Os resultados apoiam a utilização, no contexto brasileiro, do questionário PRISMA-7 como um instrumento de triagem para identificar risco de declínio funcional no idoso.

Programas de Rastreamento; Comparação Transcultural;

Questionários; Idoso 


\section{Introdução}

Manter a capacidade funcional é basilar para preservação da saúde no idoso ${ }^{1}$. Em um contexto de envelhecimento populacional sustentado, modificar a curva de declínio funcional 2, pela prevenção, assume considerável relevância. Entretanto, estudos mostram que avaliar a população idosa de forma sistemática e integralmente, utilizando escalas abrangentes, não apresenta resultados positivos e significativos. Isso decorre das inúmeras avaliações desnecessárias, realizadas em indivíduos saudáveis 3,4 . Selecionar indivíduos em risco de perda funcional para avaliação completa constitui uma estratégia mais eficaz e menos onerosa, compatível com o modelo de atenção primária à saúde 3,4,5,6.

O questionário PRISMA-7 5 foi desenvolvido no Canadá, em 2008, como parte do Modelo Assistencial PRISMA ${ }^{6}$. Trata-se de um instrumento simplificado, composto de sete itens dicótomos, destinado ao rastreamento de idosos residentes na comunidade, cujo ponto de corte de três ou mais respostas positivas identificam idosos em risco de declínio funcional.

O instrumento vem sendo utilizado pelo Royal College of General Practitioners e pela British Geriatrics Society para o rastreamento do risco de perda funcional e fragilidade 7 , amparados por estudos que mostram o bom desempenho do PRISMA-7 comparado a outros instrumentos de mesma finalidade 8,9 .

O objetivo deste trabalho foi descrever as três primeiras etapas da adaptação transcultural do PRISMA-7 para o contexto brasileiro.

\section{Métodos}

O processo de adaptação transcultural do PRISMA-7 alicerçou-se no modelo universalista, sugerido por Herdman et al. 10 (Figura 1).

O constructo de interesse, relacionado aos fatores de risco para declínio funcional, foi explorado à luz da revisão da literatura, por um grupo multidisciplinar especializado em epidemiologia e envelhecimento, que utilizou como referência a Classificação Internacional de Funcionalidade, Incapacidade e Saúde (CIF) 2, para significação dos termos autonomia; declínio funcional; mobilidade; dependência; atividade de vida diária (AVD); atividade instrumental de vida diária (AIVD) 11 e suporte social 12 .

Para a equivalência semântica 13,14 , foram realizadas duas traduções (T1/T2) do francês para o português do questionário original, de modo independente e paralelo. Essas versões foram retraduzidas para o francês, da mesma forma. A primeira tradução (T1) foi feita por uma médica fluente no idioma francês, cuja língua materna é o português e ciente dos conceitos examinados pelo questionário. A segunda tradução (T2) foi realizada por um doutor em linguística, brasileiro, sem informação sobre os conceitos e objetivos do questionário 14 , fluente na língua francesa. O objetivo da T1 foi obter uma tradução voltada à perspectiva clínica, oferecendo maior subsídio à ótica das dimensões mensuradas. Para a T2, a intenção foi refletir a utilização popular da língua fonte, captando possíveis significados ambíguos do questionário original ${ }^{14}$. As retraduções foram feitas por dois tradutores juramentados, fluentes em português, cuja língua materna é francesa, sem acesso ao instrumento original 13,14. Uma psicóloga com formação em saúde pública e em gerontologia, natural da França, radicada no Brasil e fluente nos dois idiomas, comparou o instrumento original com as traduções e retraduções, de forma mascarada, apreciando tanto o significado geral (conotativo) quanto o referencial, observando os diferentes impactos que os termos possam ter nas duas culturas 13 . Tal aspecto tem especial relevância em se tratando de funcionalidade. Algumas vezes a incapacidade para determinada tarefa é determinada mais pelas características culturais e sociais do que pela capacidade funcional em si 2,14. Na última etapa, os itens foram sistematicamente revisados pela equipe multidisciplinar, comparando o original, as traduções e retraduções, estabelecendo a versão síntese para o pré-teste 13. A apreciação do significado geral foi realizada utilizando escala com quatro categorias 13: inalterado (IN); pouco alterado (PA); muito alterado (MA); completamente alterado (CA).

Para verificar a aceitabilidade, cinco entrevistadores, sem conhecimento prévio do instrumento e com orientações simples e rápidas, aplicaram o questionário a 50 idosos, selecionados em um ambulatório de geriatria, com idade média de 72,95 $( \pm 1,45), 60 \%$ do sexo feminino e predomínio de baixa escolaridade (0 a 4 anos).

Para cada item, era solicitado que o entrevistado parafraseasse o que havia sido perguntado, para avaliar a compreensão das palavras isoladamente e o sentido geral da questão 13,14. Na impossibilidade do entrevistado escolher entre "sim" ou "não", hesitando entre "talvez", "às vezes" ou equivalente, o entrevistador deveria optar pelo "sim" como resposta 5 .

\section{Resultados}

As discussões com as equipes multidisciplinares, no Brasil e no Canadá, sugeriram que os concei- 


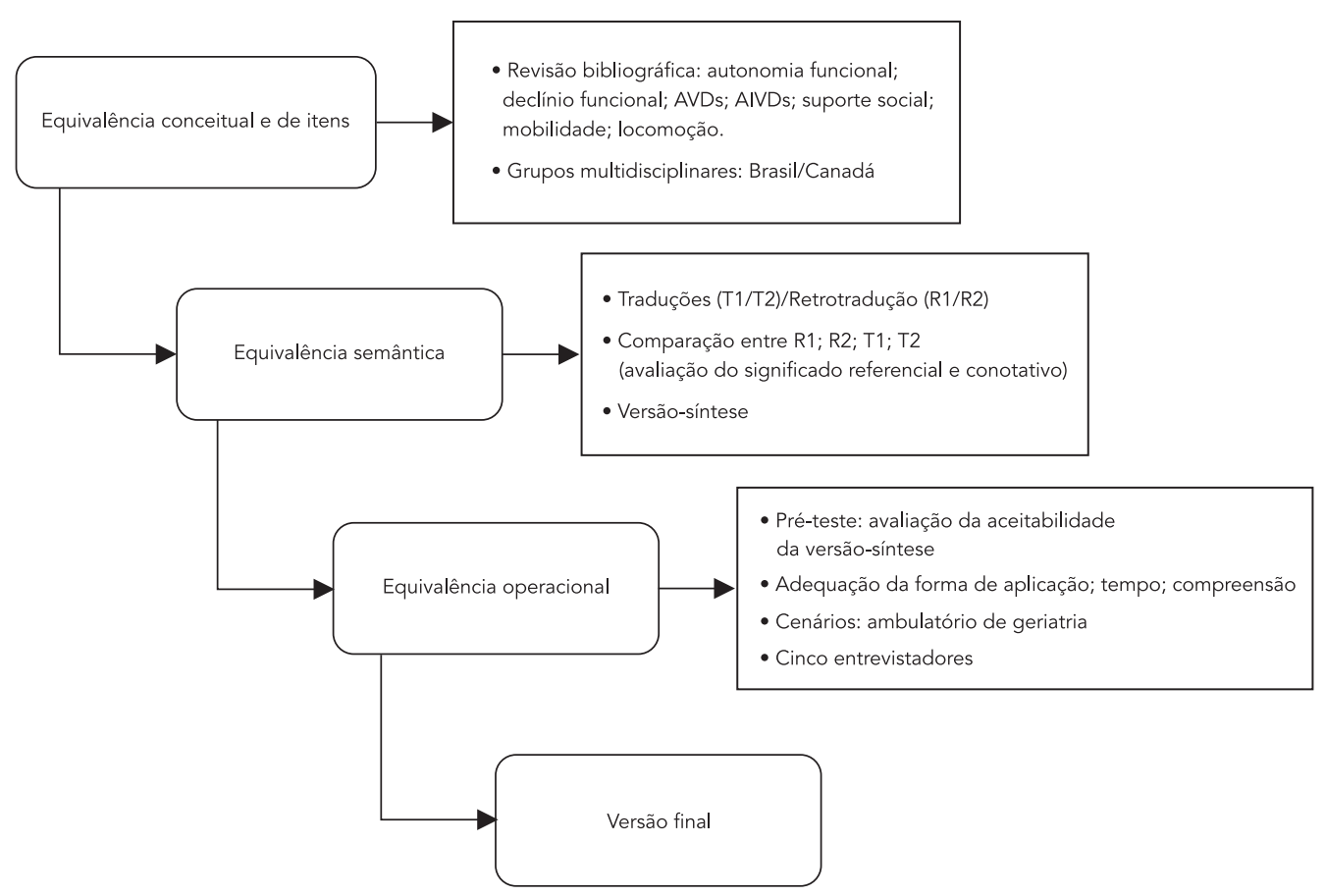

AIVD: atividade instrumental de vida diária; AVD: atividade de vida diária.

tos contidos no PRISMA-7 original, relacionados ao risco de declínio funcional 15, são pertinentes ao contexto cultural da população idosa brasileira, e seus itens apreendem as diferentes dimensões contempladas no instrumento original.

A Tabela 1 evidencia a excelente equivalência semântica entre o original e as retraduções, apresentando $71,4 \%$ dos itens com concordância integral e $28,6 \%$ entre $80 \%$ e $100 \%$. As discussões recaíram sobre os itens quatro e sete que apresentaram discrepância entre o significado do termo "frequentemente" e "regularmente". De acordo com o Dicionário Houaiss 16, na língua portuguesa, "frequentemente" pode ser utilizado no sentido de "assiduamente", isto é, de forma habitual, mas com intervalo irregular; e "regularmente" tem o sentido de "sistematicamente", com intervalo de tempo estabelecido. Na língua francesa, "régulièrement" tem como sinônimos "assidûment" e "constamment" com o sentido de assiduidade, que mais se relaciona com a base conceitual do PRISMA-7. Para a versão-síntese, a escolha recaiu sobre a expressão "regularmente", que denota a necessidade sistemática de auxílio, tanto para a questão quatro como para a sete 5 .
Optou-se pela alternativa “o senhor/senhora” ao pronome "você", visto que, frequentemente, haverá diferença etária entre o entrevistador e a população-alvo.

O pré-teste mostrou que o instrumento teve boa aceitação e foi de fácil aplicação. Todas as entrevistas foram levadas a termo em um tempo médio de três minutos com um índice de compreensão acima de $90 \%$.

Os termos "limite" e "regularmente" geraram dúvidas frequentes. Como a palavra "limite" é menos utilizada no vocabulário brasileiro, a palavra "diminua” foi agregada à questão três. Quanto ao termo "regularmente", decidiu-se por manter a mesma redação e esclarecer, quando necessário, que pode significar "diariamente" ou "todas as semanas".

Após esses ajustes, a versão final apresentada na Tabela 2 se mostrou adequada para posterior avaliação da equivalência de mensuração. 
Tabela 1

Comparação entre o original em francês e as traduções e retraduções do instrumento PRISMA-7.

\begin{tabular}{|c|c|c|c|c|c|c|c|}
\hline \multirow{2}{*}{$\begin{array}{l}\text { Original } \\
\text { 1. Avez-vous plus } \\
\text { de } 85 \text { ans? }\end{array}$} & \multicolumn{2}{|c|}{$\mathrm{T} 1 \rightarrow \mathrm{R} 1$} & \multirow{2}{*}{$\begin{array}{l}\text { SG } \\
\text { IN }\end{array}$} & \multicolumn{2}{|c|}{$\mathrm{T} 2 \rightarrow \mathrm{R} 2$} & \multirow{2}{*}{$\begin{array}{l}\text { SG } \\
\text { IN }\end{array}$} & \multirow{2}{*}{$\begin{array}{c}\text { Versão-síntese } \\
\text { O Sr(a). tem mais de } 85 \\
\text { anos? }\end{array}$} \\
\hline & $\begin{array}{c}\text { Você tem mais de } 85 \\
\text { anos? }\end{array}$ & $\begin{array}{c}\text { Tu avez plus de } 85 \\
\text { ans? }\end{array}$ & & $\begin{array}{c}\text { Você tem mais de } 85 \\
\text { anos? }\end{array}$ & $\begin{array}{l}\text { Avez-vous plus de } \\
\qquad 85 \text { ans? }\end{array}$ & & \\
\hline 2. Sexe masculin? & Sexo masculino? & Sexe masculin? & IN & Sexo masculino? & Sexe masculin? & IN & Sexo masculino? \\
\hline $\begin{array}{l}\text { 3. En général, } \\
\text { est-ce que des } \\
\text { problèmes } \\
\text { de santé vous } \\
\text { obligent à limiter } \\
\text { vos activités? }\end{array}$ & $\begin{array}{l}\text { Em geral, os problemas } \\
\text { de saúde obrigam- } \\
\text { no a limitar as suas } \\
\text { atividades? }\end{array}$ & $\begin{array}{c}\text { En général, les } \\
\text { problèmes de santé } \\
\text { vous obligent à } \\
\text { limiter vos activités? }\end{array}$ & IN & $\begin{array}{l}\text { Em geral, os problemas } \\
\text { de saúde obrigam- } \\
\text { no a limitar as suas } \\
\text { atividades? }\end{array}$ & $\begin{array}{c}\text { En général, les } \\
\text { problèmes de santé } \\
\text { l'oblige à limiter vos } \\
\text { activités? }\end{array}$ & IN & $\begin{array}{c}\text { Em geral, os problemas de } \\
\text { saúde obrigam o } \operatorname{Sr}(a) . \text { a } \\
\text { limitar suas atividades? }\end{array}$ \\
\hline $\begin{array}{l}\text { 4. Avez-vous } \\
\text { besoin de } \\
\text { quelqu'un pour } \\
\text { vous aider } \\
\text { régulièrement? }\end{array}$ & $\begin{array}{c}\text { Você precisa de alguém } \\
\text { para ajudá-lo com } \\
\text { frequência? }\end{array}$ & $\begin{array}{l}\text { Avez-vous besoin } \\
\text { de quelqu'un pour } \\
\text { vous aider avec la } \\
\text { fréquence? }\end{array}$ & MA & $\begin{array}{l}\text { Você precisa, } \\
\text { regularmente, de } \\
\text { alguém para lhe ajudar? }\end{array}$ & $\begin{array}{l}\text { Avez-vous besoin } \\
\text { quelqu'un pour aider } \\
\text { de lui régulièrement? }\end{array}$ & IN & $\begin{array}{l}\text { O Sr(a). precisa de } \\
\text { alguém para ajudá-lo } \\
\text { regularmente? }\end{array}$ \\
\hline $\begin{array}{l}\text { 5. En général, } \\
\text { est-ce que des } \\
\text { problèmes } \\
\text { de santé vous } \\
\text { obligent à rester à } \\
\text { la Maison? }\end{array}$ & $\begin{array}{l}\text { Em geral, os problemas } \\
\text { de saúde obrigam-no a } \\
\text { ficar em casa? }\end{array}$ & $\begin{array}{c}\text { En général, est-ce } \\
\text { que des problèmes } \\
\text { de santé vous } \\
\text { obligent à rester à la } \\
\text { Maison? }\end{array}$ & IN & $\begin{array}{l}\text { Em geral, os problemas } \\
\text { de saúde forçam-no a } \\
\text { permanecer em casa? }\end{array}$ & $\begin{array}{c}\text { En général, les } \\
\text { problèmes de santé } \\
\text { vous obligent à rester } \\
\text { à la maison? }\end{array}$ & IN & $\begin{array}{c}\text { Em geral, o Sr(a). tem } \\
\text { algum problema de saúde } \\
\text { que o obrigue a ficar em } \\
\text { casa? }\end{array}$ \\
\hline $\begin{array}{l}\text { 6. Pouvez-vous } \\
\text { compter sur une } \\
\text { personne que } \\
\text { vous est proche } \\
\text { em cas de besoin? }\end{array}$ & $\begin{array}{l}\text { Você pode contar } \\
\text { com alguém para } \\
\text { ajudá-lo em caso de } \\
\text { necessidade? }\end{array}$ & $\begin{array}{c}\text { Pouvez-vous avoir } \\
\text { quelqu'un tu } \\
\text { êtes près lorsque } \\
\text { nécessaire? }\end{array}$ & IN & $\begin{array}{l}\text { Você pode contar com } \\
\text { alguém para ajudá-lo } \\
\text { se necessário? }\end{array}$ & $\begin{array}{l}\text { Pouvez-vous compter } \\
\text { sur quelqu'un pour } \\
\text { l'aide si nécessaire? }\end{array}$ & IN & $\begin{array}{l}\text { Em caso de necessidade, } \\
\text { o Sr(a). pode contar com } \\
\text { alguém próximo? }\end{array}$ \\
\hline $\begin{array}{l}\text { 7. Utilisez-vous } \\
\text { régulièrement } \\
\text { une canne ou } \\
\text { une marchette } \\
\text { ou um fauteuil } \\
\text { roulant pour vous } \\
\text { déplacer? }\end{array}$ & $\begin{array}{l}\text { O senhor(a) utiliza com } \\
\text { frequência uma bengala } \\
\text { ou um andador ou uma } \\
\text { cadeira de rodas para } \\
\text { deslocar-se? }\end{array}$ & $\begin{array}{l}\text { Le seigneur (a) utilise } \\
\text { souvent une canne } \\
\text { ou une marchette ou } \\
\text { un fauteuil roulant } \\
\text { pour se déplacer? }\end{array}$ & MA & $\begin{array}{c}\text { Você utiliza } \\
\text { regularmente uma } \\
\text { bengala ou andador ou } \\
\text { cadeira de rodas para } \\
\text { locomover-se? }\end{array}$ & $\begin{array}{c}\text { Utilisez-vous } \\
\text { régulièrement } \\
\text { une canne ou une } \\
\text { marchette ou en } \\
\text { fauteuil roulant de se } \\
\text { déplacer? }\end{array}$ & IN & $\begin{array}{l}\text { O Sr(a). usa, regularmente, } \\
\text { muleta, andador ou } \\
\text { cadeira de rodas? }\end{array}$ \\
\hline
\end{tabular}

IN: inalterado; MA: muito alterado; R1: primeira retradução; R2: segunda retradução; SG: significado geral; T1: primeira tradução do inglês para o português;

T2: segunda tradução do inglês para o português.

\section{Discussão}

A base conceitual do PRISMA-7 está ancorada na CIF 2, referência para conceitos relacionados à funcionalidade, amplamente adotada tanto no Brasil quanto no Canadá.

As dimensões abordadas, que avaliam os riscos de perda funcional 17 , no instrumento original estão em consonância com os utilizados em nosso meio. A despeito disso, o grupo de especialistas ponderou que o instrumento não abrange conceitos importantes e não estabelece o grau de limitação física, funcional, de mobilidade ou locomoção.
Essas restrições se devem ao tamanho do questionário, adequado a seus objetivos. Alongar o instrumento objetivando ampliar as dimensões nele contidas levaria à perda em critérios para rastreamento ${ }^{18}$. Não identificar a intensidade do declínio se deve à finalidade do instrumento de apenas captar o risco, ficando o diagnóstico e quantificação das limitações para uma etapa posterior 5,6. Mesmo sendo consoante ao risco funcional, o item seis merece melhor avaliação na forma do questionamento. Seu comportamento ambíguo pode ser atribuído, ao menos parcialmente, às diferenças culturais entre Canadá e Brasil. No Brasil, pelas dificuldades socioe- 
Versão final do questionário PRISMA-7.

\begin{tabular}{lc}
\hline Questões & Respostas \\
\hline $\begin{array}{l}\text { 1. O Sr(a). tem mais de } 85 \text { anos? } \\
\text { 2. Sexo masculino? }\end{array}$ & Sim \\
$\begin{array}{l}\text { 3. Em geral o Sr(a). tem algum problema de saúde que o obrigue a limitar ou diminuir suas } \\
\text { atividades? }\end{array}$ & Sim \\
$\begin{array}{l}\text { 4. O Sr(a). precisa de alguém para ajudá-lo regularmente? } \\
\text { 5. Em geral, o Sr(a). tem algum problema de saúde que exija que fique em casa? }\end{array}$ & Sim \\
$\begin{array}{l}\text { 6. Em caso de necessidade, o Sr(a). pode contar com alguém próximo? } \\
\text { 7. O Sr(a). usa, regularmente, muleta, andador ou cadeira de rodas? }\end{array}$ & Sim \\
\hline
\end{tabular}

conômicas, a percepção objetiva de um suporte social pode ser considerada como positiva 12 .

A amostra selecionada para o pré-teste foi diversificada, permitindo observar que as diferenças socioculturais, de gênero, idade e escolaridade não influenciaram na compreensão das questões. O PRISMA-7 é um instrumento baseado na percepção pessoal, relacionada à funcionalidade, que pode variar, na dependência do contexto pessoal e ambiental, que influencia, em última instância, a avaliação da própria condição de saúde. Isso pode trazer discrepâncias que não interferem na capacidade de rastreamento.

\section{Colaboradores}

A. L. F. Saenger foi responsável pela integridade do estudo, pesquisa bibliográfica, conceito da investigação, delineamento do estudo, organização da coleta de dados, análise crítica e estatística dos dados e redação do artigo. C. P. Caldas foi responsável pelo conceito de estudo, definição de conteúdo intelectual e revisão crítica do conteúdo intelectual, revisão do manuscrito. L. B. Motta foi responsável pelo conceito de estudo, definição de conteúdo intelectual, e revisão do manuscrito.
Utilizando a estratégia de Herdman et al. 10, foi possível obter uma versão em português para o PRISMA-7 com uma boa equivalência conceitual, de itens e semântica. Todavia, para estabelecer um ponto de corte apropriado para o rastreamento do risco de perda funcional do idoso que vive na comunidade, no Brasil, é necessário proceder à apreciação rigorosa das propriedades psicométricas desta versão, que será objeto de próximos estudos.

\section{Agradecimentos}

Os autores agradecem a colaboração dos alunos e auxiliares de enfermagem do ambulatório de geriatria da Universidade do Grande Rio e aos idosos que se dispuseram a participar da pesquisa, colaborando para a realização deste trabalho. À equipe do Centre d'Expertise en Santé de Sherbrooke, Quebec, Canadá, pela participação nas discussões multidisciplinares, em especial à Dra. Johanne Guilbeault por todas as orientações referentes ao instrumento. 


\section{Referências}

1. Ministério da Saúde. Portaria no 2.528, de 19 de outubro de 2006. Aprova a Política Nacional de Saúde da Pessoa Idosa. Diário Oficial da União 2006; 20 out.

2. Farias N, Buchalla CM. Classificação Internacional de Funcionalidade, Incapacidade e Saúde da Organização Mundial da Saúde: conceitos, usos e perspectivas. Rev Bras Epidemiol 2005; 8:187-93.

3. Ebrahim S. Disability in older people: a mass problem requiring mass solutions. Lancet 1999; 353:1990-2.

4. Barber JH, Wallis JB. The effects of a system of geriatric screening and assessment on general practice workload. Health Bull (Edinb) 1982; 40:125-32.

5. Raîche M, Hébert R, Dubois M. PRISMA-7: a casefinding tool to identify older adults with moderate to severe disabilities. Arch Gerontol Geriatr 2008; 47:9-18.

6. Hébert R, Tourigny A, Gagnon M. Integrated service delivery ensure persons functional autonomy. Quebec: Edisem Inc.; 2005.

7. Turner G, Clegg A. Best practice guidelines for the management of frailty: a British Geriatrics Society, Age UK and Royal College of General Practitioners report. Age Ageing 2014; 43:744-7.

8. Hébert R, Raîche M, Dubois M, Gueye NR, Dubuc $\mathrm{N}$, Tousignant $\mathrm{M}$, et al. Impact of PRISMA, a coordination-type integrated service delivery system for frail older people in Quebec (Canada): quasiexperimental study. J Gerontol B Psychol Sci Soc Sci 2010; 65B:107-18.

9. Hoogendijk EO, van der Horst HE, Deeg DJH, Frijters DH, Prins BA, Jansen AP, et al. The identification of frail older adults in primary care: comparing the accuracy of five simple instruments. Age Ageing 2013; 42:262-5.
10. Herdman M, Fox-Rushby J, Badia X. Model of equivalence in the cultural adaptation of HRQoL instruments: the universalist approach. Qual Life Res 1998; 7:323-35.

11. Lino VTS, Pereira SRM, Camacho LAB, Ribeiro Filho ST, Buksman S. Adaptação transcultural da Escala de Independência em Atividades da Vida Diária (Escala de Katz). Cad Saúde Pública 2008; 24:103-12.

12. Gonçalves TR. Avaliação de apoio social em estudos brasileiros: aspectos conceituais e instrumentos. Ciênc Saúde Coletiva 2011; 16:1755-69.

13. Reichenheim M, Moraes CL. Operacionalização de adaptação transcultural de instrumentos de aferição usados em epidemiologia. Rev Saúde Pública 2007; 41:665-73.

14. Beaton DC, Bombardier C, Guillemin F, Feraz MB. Guidelines for the process of cross-cultural adaptation of self-report measures. Spine (Phila $\mathrm{Pa}$ 1976) 2000; 25:3186-91.

15. World Health Organization. International classification of functioning, disability and health: ICF. Geneva: World Health Organization; 2001.

16. Instituto Antônio Houaiss. Dicionário Houaiss da língua portuguesa. Rio de Janeiro: Editora Objetiva; 2001.

17. Robichaud L, Hébert R, Roy C. A preventive program for community-dwelling elderly at risk of functional decline: a pilot study. Arch Gerontol Geriatr 2000; 30:73-84.

18. Gray JA. New concepts in screening. Br J Gen Pract 2004; 54:292-8. 


\section{Abstract}

Screening with a simplified instrument is effective for identifying elderly at risk of functional decline, for more comprehensive subsequent evaluation. However, use of a measurement tool in a different sociocultural context from the original requires prior adaptation. The current article aims to describe the initial stages of a cross-cultural adaptation of PRISMA-7, a questionnaire developed to identify community-dwelling elderly at risk of functional decline. Evaluation of conceptual and item equivalences showed pertinence to the Brazilian context. Semantic equivalence covered the correspondence in referential (denotative) and connotative meaning. The pretest showed that the instrument's synthesis version has good acceptability. The results suggest the use of PRISMA-7 in the Brazilian context as a screening tool for identifying risk of functional decline in the elderly.

Mass Screening; Cross-Cultural Comparison; Questionnaires; Aged

\section{Resumen}

El rastreo es una manera eficaz de localizar ancianos en riesgo de declive funcional. Con este fin, es necesario un instrumento simplificado, capaz de identificar a estos individuos para una evaluación posterior más amplia. No obstante, la utilización de un instrumento de medida en un contexto sociocultural diferente de aquel para el que fue desarrollado, requiere una adaptación previa. Este artículo tiene por objetivo describir las primeras etapas del proceso de adaptación transcultural del PRISMA-7, cuestionario desarrollado para identificar ancianos que viven en la comunidad y se encuentran en riesgo de pérdida funcional. Las evaluaciones de las equivalencias, conceptuales y de items mostraron pertinencia en el contexto brasileño. La equivalencia semántica contempló la correspondencia del significado referencial (denotativo) y del significado connotativo. El pre-test demostró que la versión-síntesis del instrumento tiene una buena aceptabilidad. Los resultados apoyan la utilización, en el contexto brasileño, del cuestionario PRISMA-7 con un instrumento de cribado para identificar riesgo de declive funcional en el anciano.

Tamizaje Masivo; Comparación Transcultural; Cuestionarios; Anciano
Recebido em 06/Mai/2015

Versão final reapresentada em 22/Jan/2016 Aprovado em 23/Fev/2016 